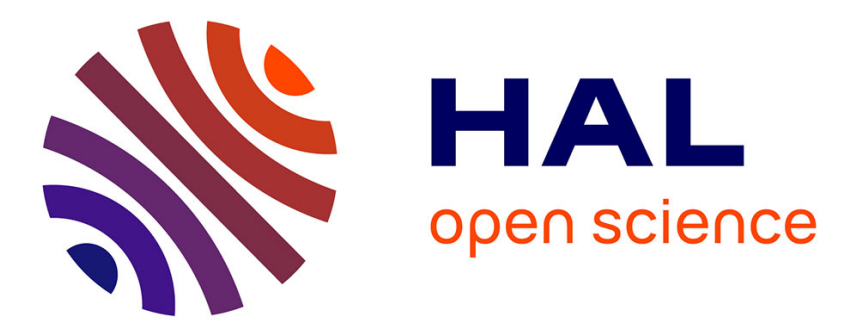

\title{
Protocol for Recording Enamel Hypoplasia in Modern and Archaeological Caprine Populations
}

Bethan Upex, Marie Balasse, Anne Tresset, Benjamin Arbuckle, Keith Dobney

\section{To cite this version:}

Bethan Upex, Marie Balasse, Anne Tresset, Benjamin Arbuckle, Keith Dobney. Protocol for Recording Enamel Hypoplasia in Modern and Archaeological Caprine Populations. International Journal of Osteoarchaeology, 2012, pp.doi 10.1002/oa.2227. 10.1002/oa.2227 . hal-00745003

\section{HAL Id: hal-00745003 https://hal.science/hal-00745003}

Submitted on 24 Oct 2012

HAL is a multi-disciplinary open access archive for the deposit and dissemination of scientific research documents, whether they are published or not. The documents may come from teaching and research institutions in France or abroad, or from public or private research centers.
L'archive ouverte pluridisciplinaire HAL, est destinée au dépôt et à la diffusion de documents scientifiques de niveau recherche, publiés ou non, émanant des établissements d'enseignement et de recherche français ou étrangers, des laboratoires publics ou privés. 


\author{
*** Manuscript published in International Journal of Osteoarchaeology (2012) *** \\ Published online in Wiley Online Library \\ (wileyonlinelibrary.com) DOI: 10.1002/oa.2227
}

\title{
PROTOCOL FOR RECORDING ENAMEL HYPOPLASIA IN MODERN AND ARCHAEOLOGICAL CAPRINE POPULATIONS
}

\author{
Bethan Upex ${ }^{a}$; Marie Balasse ${ }^{\mathrm{b}}$; Anne Tresset ${ }^{\mathrm{b}}$; Benjamin Arbuckle ${ }^{\mathrm{c}}$; \\ Keith Dobney ${ }^{\text {a }}$
}

\begin{abstract}
a University of Aberdeen, Department of Archaeology, Elphinstone Road, Aberdeen, AB24 3UF, UK. b Muséum national d'Histoire naturelle, CNRS UMR 5197, Archéozoologie, Archéobotanique : Sociétés, Pratiques et Environnements, Département Écologie et Gestion de la Biodiversité, CP 56, 55 rue Buffon, F-75231 Paris cedex 05

c Baylor University, Department of Anthropology, Forensic Science, \& Archaeology, One Bear Place \#97173, Waco TX 76798-7173, USA.
\end{abstract}

\begin{abstract}
This paper outlines the first methodology for recording dental enamel hypoplasia in the highcrowned dentition of modern and archaeological caprine teeth. The method has been developed and trialed on five caprine populations from Orkney (UK); two modern populations (Shetland and North Ronaldsay breeds) and three Neolithic assemblages from the archaeological sites of Knap of Howar, Skara Brae and Holm of Papa Westray. Problems associated with differential tooth wear, as well as the presence of coronal cementum are discussed, and recommendations are given on the identification and recording of hypoplastic dental defects in caprines.
\end{abstract}

\section{INTRODUCTION}

Enamel hypoplasia is a commonly occurring dental defect that is widely considered to be a good indicator of physiological stress occurring during dental crown development. Of the various forms of enamel defects that can occur, dental enamel hypoplasia can be simply defined as a deficiency in the thickness of the dental enamel (Goodman \& Rose, 1990). Dental enamel hypoplasias have been used in many archaeological, anthropological and zoological studies to reveal aspects of dietary deficiencies and general health in past human and animal populations (e.g. Goodman, et al. 1989; Dobney \& Ervynck, 1998; 2000; King, et al. 2005; Oyamada, et al. 2008).

While it has been suggested that microscopic analysis of tooth thin sections is the only sure way to accurately identify all enamel hypoplasia, this obviously not practical on a large scale (Kierdorf, et al. 2006). Therefore specific methodologies for the macroscopic recording of enamel hypoplasia have been developed. These methodologies have largely focused on lowcrowned species such as pigs (Dobney \& Ervynck, 1998) and humans (FDI 1982). However, these methodologies are difficult to apply to high-crowned species due to a number of problems. These include: 1) the rate of enamel attrition and its removal of the upper portions of the tooth crown; 2) the position of the tooth crown in the mandibular bone causing most of it to be hidden; and 3) the presence of coronal cementum covering the enamel surface and potentially obscuring enamel defects. Despite these problems some of the earliest studies of enamel hypoplasia in archaeological non-primate animal populations were conducted on domestic caprine teeth (Gifford-Gonzalez, 1984; 1985; Marshall, 1990). These studies, performed on Neolithic assemblages from East Africa, provided valuable data on the 
frequency of enamel hypoplasia in caprines and discussed their significance in terms of the reconstruction of former breeding strategies. Building on this work, Arbuckle (unpublished) and Balasse et al. (2010) provided the first evidence that enamel hypoplasia may occur frequently on the teeth of modern and archaeological caprine populations and can easily be identified. Enamel hypoplasia has also been investigated in other high-crowned species (such as giraffe and other African fossil large herbivores: Franz-Odendaal, et al., 2003; FranzOdendaal 2004; and bison: Niven, et al. 2004; Byerly, 2007, see Kierdorf, et al. 2006 for a critique of this work) using methods based on the Fédération Dentaire Internationale (FDI, 1982), Ensor and Irish (1995) and Dobney and Ervynck (1998). However, none of the above studies addresses problems of dental wear and coronal cementum, or uses a systematic strategy for recording of enamel hypoplasia in high-crowned species.

Clearly there is potential for enamel hypoplasia to be used to address a variety of zooarchaeological questions, but in high-crowned species this potential is limited by the difficulties in recording enamel hypoplasia. This paper attempts to bridge this gap by developing a systematic methodology, and by assessing the difficulties faced in recording enamel hypoplasia in species with high-crowned teeth. While this study focuses on recording enamel hypoplasia in sheep, the methodology developed can be applied to other species with high dental crowns, such as goats or cattle. The methodology presented here has been adapted from that developed on low-crowned teeth (Dobney \& Ervynck, 1998; FDI, 1982) and the first interpretive results from the material used in this paper will be published shortly (Upex, et al. in prep; Upex \& Dobney, in prep).

\section{MATERIAL}

The methodology is trialled on five sheep populations of both modern and archaeological origin derived from the Orkney archipelago, located off the northernmost tip of Scotland. Modern material is represented by 85 mandibles from sheep of the North Ronaldsay (NR) breed, sourced from the island of North Ronaldsay, and 34 Shetland sheep mandibles from the island of Hoy. The archaeological material consists of isolated mandibular teeth from the sites of Knap of Howar, a settlement on the island of Papa Westray dating to the earliest known occupation of the archipelago c. 3500 cal BC (Sheridan \& Higham 2006; 2007) (KH; n = 77); Skara Brae, a settlement on the Orkney Mainland dating between $3360-2440 \mathrm{cal} \mathrm{BC}$ (Pers. com. D. Clark and A. Shepherd) (SB; $\mathrm{n}=62$ ); and Holm of Papa Westray North, a chambered tomb on the small island located just off Papa Westray that gave the site its name, with animal remains dating between 2880 - $2470 \mathrm{cal} \mathrm{BC}$ (Ashmore 2009) (HPWN; $\mathrm{n}=57$ ).

\section{METHODOLOGY}

\section{Which teeth?}

Physiological or environmental stress events resulting in enamel hypoplasia should theoretically affect all developing teeth. The decision was taken to focus on permanent mandibular molars, for the following reasons:

1) There is considerably more data to support the accurate aging, eruption and wear of mandibular teeth than is available for maxillary teeth (e.g. Silver, 1970; Payne, 1973). This information is essential for the reconstruction of the timing of hypoplasia formation.

2) The three permanent molars contain a continuous record of approximately the first 30 months of an individual's life, while the incisors and permanent premolar crowns develop in much shorter time periods (Weinreb and Sharav, 1964) and would therefore provide partial or redundant information. Work by Witter and Mizek (1999) indicates that the lower first molar (M1) starts growing in utero approximately three months before birth. Upex and Dobney (in prep) have demonstrated that in modern animals from Orkney, the first molar is completed around 8 months after birth; development of the crown of the lower second molar (M2) starts in the second month of life and is 
completed approximately 16 months after birth; the development of the lower third molar crown (M3) is more variable but begins development around 15 months and is completed by approximately 30 months after birth.

3) Sheep deciduous teeth develop very rapidly (with deciduous lower premolars developing over approximately 3.5 months: Witter and Mizek, 1999), limiting the time period in which stress events leading to enamel hypoplasia formation can be recorded. Also, because deciduous teeth form in utero (Weinreb and Sharav, 1964) they are to some extent buffered by the mother from external stresses. Work by one of us (Arbuckle, unpublished), on caprines from the Neolithic central Anatolian sites of Çatalhöyük and Erbaba, demonstrated very low numbers of hypoplasia recorded in deciduous teeth (as well as on the permanent pre-molars). This pattern was also found in studies of modern and extinct populations of Giraffidae (Franz-Odendaal, et al. 2003; Franz-Odendaal, 2004).

\section{Tooth extraction}

Perhaps the most obvious problem with recording enamel hypoplasia in species with highcrowned teeth is the fact that a large part of the tooth is buried within the alveolus, making enamel defects impossible to see. In fragmented archaeological material this can be overcome by using isolated teeth, although this then causes problems with the separation of the first and second molars. In the complete mandibular tooth rows from the modern material used in this study, the complete tooth was removed from the bone. This was done by carefully cutting away the lingual surface of the mandibular corpus in three sections (one over each of the permanent molars), joined by a running cut along the canalis mandibulae (see Figure 1). The lingual mandibular surface was chosen over the buccal as the bone is thinner and therefore easier to remove.

\section{Removing dental cementum}

Dental cementum provides a firm attachment for the periodontal ligaments and holds the tooth in its socket. In species with low-crowned teeth, cementum forms only on the roots. However, in species with high-crowned teeth, cementum also covers the enamel surface, anchoring the tooth into the bone while the root develops and the tooth erupts into the mouth. Cementum on high-crowned teeth can cause a number of problems in recording enamel hypoplasia, most obviously because it covers the enamel surface, potentially obscuring defects (Kierdorf, et al. in prep, see Figure 2). Cementum is deposited in layers that are laid down periodically in response to continued tooth eruption. Variation in these 'imbricational' cementum layers can result in the formation of a ridged surface that mimics enamel hypoplasia (Kierdorf, et al. 2006). Where cementum was observably present and probably obscuring hypoplasia visibility, it was removed from the enamel surface by gently drilling using a tungsten drill bit. As cementum is softer that dental enamel it was easy to differentiate between the two whilst drilling, and therefore possible to remove the cementum without damaging the enamel surface.

\section{Dental wear}

Dental wear is a potential complication in the assessment of enamel defects in high-crowned species. Dental wear removes the upper portion of tooth crown along with any enamel hypoplasia occurring there. In order to test the impact of dental wear on the recording of enamel hypoplasia, complete mandibles from the modern samples were recorded using Payne's (1973) age-categories C (6 - 12 months) through to F (3-4 years) (see Figure 3$)$. Before Payne age C (6-12 months), mineralization of the first molar is incomplete, making the identification of enamel hypoplasia difficult, and from age-category G (4 - 6 years) onwards, dental wear may become extensive, with the potential removal of large portions of the tooth crown. In the archaeological populations, teeth in all stages of dental wear were recorded, due to the difficulties of assigning isolated teeth to specific age-categories.

\section{Left or right?}


The decision to focus on either left or right-sided specimens is variable between assemblages. In the Hoy population both left and right sides of the mandible were available for study; however, only one side was selected to minimize the duplication of data. The side selected depended on the preservation, dental wear and location of pathologies. Similar factors were considered for the NR material. As the archaeological assemblages used in this study consisted of isolated teeth from mixed butchery refuse and natural accumulations, both left and right sides were recorded, as there was little chance of reuniting teeth from the same individual.

\section{Defect types}

Teeth were viewed under a strong oblique light to allow the enamel hypoplasia to be seen more clearly. As enamel hypoplasias most commonly take the form of a groove or line running horizontally across the tooth surface, or of single / multiple pits in the enamel, the defects are revealed as enhanced shadows on the enamel surface when light is shone vertically and obliquely across the tooth surface. Once defects were located and positively identified, they were recorded as a 'line', 'depression' or 'pit' based on the descriptions of Dobney et al. (1998) and the Fédération Dentaire Internationale (FDI, 1982) (See Figure 4). Work by Witzel et al. $(2006 ; 2008)$ in pigs and humans has suggested that these different defect types relate to differences in the intensity and/or duration of the stress episode.

\section{Which surface to record?}

Unpublished work by one of us (BA) has demonstrated that in caprines, enamel hypoplasia is consistently more common on the buccal surface of the tooth crown, and that where hypoplasias do occur on the lingual surface, it is almost always in relation to a more visible defect on the buccal surface (a pattern repeated in the Orkney datasets, see Figure 6). In the caprine populations studied by BA this pattern is most obvious in the first molars where enamel hypoplasias were identified almost three times as often on the buccal compared to the lingual surface. This pattern is also evident in the M2 and M3 but the differences are of a smaller scale. Similar observations are reported by Niven et al. (2004) in bison lower molars. This is a noticeable difference with methodologies developed for low crowned pig teeth, for example, where enamel hypoplasia is recorded on the lingual surface of the molars where the crown is the highest, rendering the lines more obviously separated and more readily recorded (Dobney \& Ervynck, 1998). In sheep teeth, the lower visibility of linear hypoplasia on the lingual surface is mostly likely due to a thinner enamel layer on the lingual side of the tooth, leading to shallower, less obvious defects. Because the lingual surface showed fewer enamel hypoplasias, only the buccal surface was recorded in this study.

\section{Measurements}

In incomplete tooth crowns the pitted and porous appearance of the unmineralised enamel can render the accurate identification of enamel hypoplasia very difficult (Figure 5). However, in sheep, the permanent molars erupt into the mouth and come into wear before they have completed their crown development, meaning that in complete teeth, the upper portion of the crown has already been lost due to dental wear. Therefore, it is important to assess unworn incomplete teeth for enamel hypoplasia in the upper portions of the tooth crown. Enamel hypoplasia was recorded in incomplete unworn teeth by measuring down the centre of each lobe of the buccal surface from the tip of each cusp to the enamel defect (see Figure 5). While these measurements cannot be combined with the measurements of hypoplasia taken on complete teeth, they provide an important record of enamel defects occurring in the upper portions of the teeth, before the REJ is complete and dental wear has begun.

Because enamel hypoplasia is potentially lost through tooth wear, it is necessary to record how much of the remaining tooth crown could be observed. In incomplete teeth a simple measurement of crown height was taken (shown in Figure 5). Measurements of tooth crown height in complete teeth were taken on the lingual surface, from the groove between the tooth roots to the apex of the anterior cusp (Figure 6). The groove between the roots on the lingual 
surface provides a constant point on all teeth from which to measure, overcoming any problems associated with locating a consistent reference point on the variable root-enamel junction (REJ). Measurements were taken to the nearest $0.1 \mathrm{~mm}$ with digital calipers. The location of enamel hypoplasia was recorded on each lobe, by measuring up the centre of each lobe from the REJ to the defect (Figure 6). In cases where the defect took the form of a large pit, cluster of small pits, or a wide groove, the central point of the defect was recorded.

\section{RESULTS}

Hypoplasia occurrence and type

Enamel hypoplasias were identified in all 5 populations and are summarized in Table 1. Enamel hypoplasias were far more common in teeth classed as complete (with REJ present) compared with incomplete teeth. All three defect types were identified in all 5 study populations (shown in Table 2). Linear and depression type defects were the most common, with only a very limited number of pit type defects identified.

\section{Cementum}

None of the complete teeth from the modern populations had thick deposits of cementum, meaning that hypoplasia was visible through the cementum (see Figure 6). However, all of the complete teeth from KH, $10(16 \%)$ of the complete teeth from SB, and all of the complete teeth from HPWN had thick cementum layers that were removed, revealing the enamel surface beneath. In 4 of the 10 teeth from SB, the removal of cementum revealed hypoplasias that were previously not visible.

\section{Measurements and dental wear}

As shown in Figure 3 and 7 (for the modern datasets), dental wear in the first 4 years of life removes between 6 to 19 millimeters of tooth crown on the 1 st molar; 2 to 10 millimeters on the 2 nd molar and the same on the 3 rd molar in these populations. Figure 8 shows the location of hypoplasia in the modern datasets on the anterior, posterior and central lobes of the three permanent molars. This figure also maps the dental wear in each population, showing the total crown heights present, in relation to the location of the enamel hypoplasia. The archaeological data is presented in this way in Figure 9.

\section{DISCUSSION AND CONCLUSION}

How practical is this methodology?

Once teeth had been extracted, they could be recorded by one person at a rate of approximately 100 teeth per day, allowing large amounts of systematic data to be gathered. Unfortunately, the process of tooth extraction from complete mandibles takes longer, with one person being able to extract the teeth from approximately 25 mandibles per day. The speed at which this could be done is also highly variable, depending on the state of preservation of the sample. For example, modern material with high collagen content took longer as the bone was less friable, whereas archaeological samples could potentially be extracted very quickly due to the more friable and brittle nature of the bone. The principal drawback to the methodology is obviously the destructive nature of the extraction process, something that may need to be considered carefully for important or rare specimens, or collections.

\section{What problems are faced in recording the data using this method?}

There are a number of problems associated with the implementation and repeatability of systematic recording protocols, and the method outlined here is no exception. The main problem identified was that: as observations were made with the naked eye, it was difficult to verify systematically the presence of the most minor linear types of dental defect, given their sliding scale from the macro- to the microscopic. It was possible that some defects were 
overlooked, particularly the more minor depression and linear type defects. The use of strong oblique lighting helped to overcome this problem. The use of a hand lens or retractable lens may also ensure that all defects are recorded, although the use of magnification was not adopted in this study. If there was doubt, it was often found useful to re-record the tooth at a later date and check the status and measurements of defects that had originally been recorded. Re-recording was carried out on two occasions on the North Ronaldsay population in order to check the repeatability of the defect identification, and all of the defects recorded in the original study were identified in the subsequent runs.

\section{Dealing with coronal cementum and dental wear}

Coronal cementum is clearly a problem in the analysis of enamel hypoplasia in caprine populations, although it is not insurmountable. While cementum covers all teeth, it varies in its thickness between populations, and in teeth where it is thick it can be removed carefully to reveal hidden enamel hypoplasia.

In the first 4 years of life approximately $20 \%$ of the total tooth height is lost to dental wear in the four Payne age classes recommended for study $(\mathrm{C}=6-12$ months; $\mathrm{D}=1-2$ years; $\mathrm{E}=2-3$ years and $\mathrm{F}=3-4$ years). Figures $8 \& 9$ clearly demonstrate that in all 5 samples there is adequate representation of the upper portions of the tooth crowns but that enamel hypoplasias are absent from them, occuring almost exclusively in the cervical portion of the tooth crown, regardless of state of dental wear. There are several possible explanations, including changes in the internal structure of the tooth and the angle of Striae of Retzius for the consistent occurrence of enamel hypoplasia in the cervical portion of the tooth crown. However, the most likely cause is variation in crown extension rates. Kierdorf (et al. in prep) suggest that formation of the cervical $25 \%$ of crown takes up approximately $50 \%$ of the total crown formation period. This is supported by the low frequency of enamel hypoplasia in incomplete teeth.

\section{Conclusions}

This study has outlined a methodology for recording enamel hypoplasia in caprine teeth. It has demonstrated that enamel hypoplasia is both common and easily recordable in modern and archaeological caprine populations, and that the two main problems associated with the study of dental defects in species with high-crowned teeth are not insurmountable. While the presence of coronal cementum can be problematic it can also be removed, revealing the enamel surface. Dental wear in caprine populations does not pose a real problem to the accurate recording of enamel defects. This study has demonstrated that the amount of enamel lost to dental wear in the first 4 years of life can be up to approximately $20 \%$ of the total tooth height, but that regardless of dental wear, enamel hypoplasia occurs almost exclusively in the cervical portion of the tooth crown. Consequently, as long as there is adequate representation of the younger age categories in each population, there is little chance that recording teeth in more advanced wear stages will significantly bias the location of enamel hypoplasia to the lower half of the crown. This lack of hypoplasia in the upper portions of the tooth crown explains the very limited number of defects recorded in incomplete molars, suggesting that the recording of these teeth may not be very informative, and has been linked to variation in enamel growth rates down the tooth crown. Clearly the location of enamel defects almost entirely in the cervical portion of the tooth crown has implications for future studies of enamel hypoplasia in caprines, particularly with regard to work linking the timing and location of hypoplastic events to known physiological or seasonal stress events.

\section{ACKNOWLEDGEMENTS}

We would like to thank several people for their assistance with this paper: Professor Horst Kierdorf, Dr Uwe Kierdorf and Dr Carston Witzel from the University of Hildesheim for allowing us to use their thin section image and photographs, and for their helpful comments 
on the text; Professor Terry O'Connor, for allowing us to work on the material from Hoy; National Museums Scotland particularly Drs Alison Sheridan, David Clarke and Andrew Kitchener, for providing permission to study the archaeological material from Skara Brae, Knap of Howar and Holm of Papa Westray North. Thanks also go to the University of Durham for partly funding this work through their Durham Doctoral Fellowship scheme. This work was also supported by an INSU CNRS ECLIPSE II 2005-2007 program (Climatic constraints and development of Neolithic husbandry in Western Europe at the Atlantic / SubBoreal boundary (4 millennium BC) dir. M. Balasse and A. Tresset) and an ERC Starting Grant GA 202881 SIANHE (dir. M. Balasse).

\section{BIBLIOGRAPHY}

Arbuckle, B. Unpublished manuscript. Enamel defects in sheep and goat teeth: Possibilities for examining developmental stress in archaeological caprine populations'

Ashmore, P. 2009. Radiocarbon dates. In: Ritchie, A. (Ed.) On the fringe of Neolithic Europe. Excavation of a chambered cairn on the Holm of Papa Westray, Orkney. Edinburgh, Society of Antiquaries of Scotland: 59-65.

Balasse, M. and Tresset, A. 2007. Environmental constraints on the reproductive activity of domestic sheep and cattle: what latitude for the herder? Anthropozoologica 42(2): 71-88.

Balasse, M. and Tresset, A. 2009. A key to the adaption of the Neolithic husbandry in the Orkneys: contribution of seaweed to the sheep diet at the Holm of Papa Westray, revealed through stable isotope analysis $\left(\delta^{13} \mathrm{C}\right.$ and $\left.\delta^{18} \mathrm{O}\right)$ of teeth. In: Ritchie, A. (Ed.) On the fringe of Neolithic Europe. Excavation of a chambered cairn on the Holm of Papa Westray, Orkney. Edinburgh, Society of Antiquaries of Scotland : 74-83.

Balasse, M. Upex, B. and Ambrose, S. 2010. The influence of environmental factors on enamel hypoplasia in domestic sheep and goats in southern Kenya, Masailand. Documenta Archaeobiologiae 7: 3 - 13.

Byerly, RM. 2007. Palaeopathology in late Pleistocene and early Holocene Central Plains bison: dental enamel hypoplasia, fluoride toxicosis and the archaeological record. Journal of Archaeological Science 34(11): 1847-1858.

Dobney, KM. and Ervynck, A. 1998. A Protocol for recording linear enamel hypoplasia on archaeological pig teeth. International Journal of Osteoarchaeology 8: 263 - 273.

Dobney, KM. and Ervynck, A. 2000. Interpreting developmental stress in archaeological pigs: The chronology of linear enamel hypoplasia. Journal of Archaeological Science 27: 597 607.

Ensor, BE. and Irish, JD. 1995. Hypoplastic area method for analyzing dental enamel hypoplasia. American Journal of Physical Anthropology 98: 507-517.

F. D. I. 1982. An epidemiological index of developmental defects of dental enamel (DDE Index). International Dental Journal 32 (Technical Report 16): 159-167.

Franz-Odendaal, T. 2004. Enamel hypoplasia provides new insights into early systemic stress in wild and captive giraffes (Giraffa camelopardalis). Journal of Zoology (London) 263: 197-206.

Franz-Odendaal, T. Lee-Thorp, JA. and Chinsamy, A. 2003. Insights from stable light isotopes on enamel defects and weaning in Pliocene herbivores. Journal of Bioscience 28(6): 765-773.

Goodman, AHC. Martinez, DL. Perry, A. Martinez, C. Chavez, A. and Dobney, K. 1989. Nutritional supplementation and the development of linear enamel hypoplasia in children from Tezonteopan. American Journal of Clinical Nutrition. 53: 773-781.

Goodman, AH. and Rose, JC. 1990. Assessment of systemic physiological perturbations from dental enamel hypoplasia and associated histological structures. Yearbook of Physical Anthropology 33: 59 - 110.

Kierdorf, H. Witzel, C. Upex, B. Dobney, K. and Kierdorf, U. In prep. Enamel hypoplasia in molars of sheep and goats as related to tooth crown formation and enamel structure. Archives of Oral Biology. 
Kierdorf, H. Zeiler, J. and Kierdorf, U. 2006. Problems and pitfalls in the diagnosis of linear enamel hypoplasia in the cheek teeth of cattle. Journal of Archaeological Science 33: 1690 - 1695.

King, T. Humphrey, LT. and Hillson, S. 2005. Linear enamel hypoplasia as indicators of systemic physiological stress: evidence from two known age-at-death populations from post-medieval London. American Journal of Physical Anthropology 128: 547 - 559.

Milhaud G. and Nézit J. 1991. Développement des molaires chez le mouton. Etude morphologique, radiographique et microdurométrique. Recueil de Médecine Vétérinaire 167: 121-127.

Niven, L. Egeland, CP. and Todd, LC. 2004. An inter-site comparison of enamel hypoplasia in bison: implications for paleoecology and modeling Late Plains Archaic subsistence. Journal of Archaeological Science 31: 1783 - 1794

Oyamada, J. Igawa, K. Kitagawa, Y. Manabe, Y. Kato, K. Matsushita, T. and Rokutanda, A. 2008. Pathology of deciduous teeth in the samurai and commoner children of early modern Japan. Anthropological Science 116 (1): 9 -15

Payne, S. 1973. Kill-off patterns in sheep and goats. The mandibles from Asvan Kale. Anatolian Studies 23: 281-303.

Ritchie, A. 1983. Excavations of a Neolithic farmstead at Knap of Howar, Papa Westray, Orkney. Proceedings of the Society of Antiquaries of Scotland 113: 40-121.

Schulting, R. and Richards MP. 2009. Radiocarbon dates and stable isotope values on human remains. In: Ritchie, A. (Ed.) On the fringe of Neolithic Europe. Excavation of a chambered cairn on the Holm of Papa Westray, Orkney. Edinburgh, Society of Antiquaries of Scotland: 66-73.

Sheridan A. and Higham T. 2006. The re-dating of some Scottish specimens by the Oxford Radiocarbon Accelerator Unit (ORAU). Discovery and Excavations in Scotland 7: 202209.

Sheridan A. and Higham T. 2007. The re-dating of some Scottish specimens by the Oxford Radiocarbon Accelerator Unit (ORAU): results received during 2007. Discovery and Excavations in Scotland 8: 225.

Silver, IA. 1970. The ageing of domestic animals. In: Brothwell, D. and Higgs, E. (Eds) Science in Archaeology. A survey of progress and research. New York, Praeger Publishers: 283-302.

Tresset, A. 2003. French Connections II: of cows and men. In: Armit, A. Murphy, E. Nelis, EL and Simpson DDA. (Eds) Neolithic Settlement in Ireland and western Britain. Oxford, Oxbow, 18-30.

Upex, B. Balasse, M. Treset, A. and Dobney, K. In prep. Archaeological applications of Enamel Hypoplasia: an interpretive framework for understanding climatic and environmental adaptations. Journal of Archaeological Science.

Upex, B. and Dobney. K. In prep. Dental enamel hypoplasia as indicators of physiological and seasonal stress in modern sheep populations. Journal of Zoology.

Weinreb, MM. and Sharav, DMD. 1964. Tooth development in sheep. American Journal of Veterinary Research 25(107): 891-908.

Witter, K. and Misek, I. 1999. Time programme of the early tooth development in the domestic sheep (Ovis aries, Ruminantia). Acta Veterinaria Brno 68: 3-8.

Witzel. C. Kierdorf, U. Dobney, K. Ervynck, A. Vanpoucke, S. and Kierdorf, H. 2006. Reconstructing impairment of secretory ameloblasts function in porcine teeth by analysis of morphological alterations in dental enamel. Journal of Anatomy 209: 93 - 110

Witzel, C. Kierdorf, U. Schults, M. and Kierdorf, H. 2008. Insights from the inside: histological analysis of abnormal microstructure associated with hypoplastic enamel defects in human teeth. American Journal of Physical Anthropology 136: 400- 41 
Table 1: Summary of the enamel hypoplasia recorded in complete and incomplete teeth in the five study populations.

\begin{tabular}{|c|c|c|c|}
\hline Complete teeth & $\begin{array}{l}\text { Number of teeth } \\
\text { recorded }\end{array}$ & $\begin{array}{l}\text { Number of teeth } \\
\text { with hypoplasia }\end{array}$ & $\begin{array}{c}\text { Percentage of } \\
\text { teeth with } \\
\text { hypoplasia }\end{array}$ \\
\hline North Ronaldsay & 147 & 116 & $79 \%$ \\
\hline Hoy & 76 & 46 & $60 \%$ \\
\hline Knap of Howar & 77 & 38 & $49 \%$ \\
\hline Skara Brae & 62 & 27 & $43 \%$ \\
\hline $\begin{array}{l}\text { Holm of Papa } \\
\text { Westray }\end{array}$ & 57 & 16 & $28 \%$ \\
\hline Incomplete teeth & $\begin{array}{c}\text { Number of teeth } \\
\text { recorded }\end{array}$ & $\begin{array}{l}\text { Number of teeth } \\
\text { with hypoplasia }\end{array}$ & $\begin{array}{l}\text { Percentage of } \\
\text { teeth with } \\
\text { hypoplasia }\end{array}$ \\
\hline North Ronaldsay & 45 & 4 & $9 \%$ \\
\hline Hoy & 9 & 1 & $11 \%$ \\
\hline Knap of Howar & 48 & 7 & $15 \%$ \\
\hline Skara Brae & 28 & 3 & $11 \%$ \\
\hline $\begin{array}{l}\text { Holm of Papa } \\
\text { Westray }\end{array}$ & 21 & 1 & $5 \%$ \\
\hline
\end{tabular}


Table 2: Comparing the number of hypoplasia of each type across the 5 study populations

\begin{tabular}{|c|c|c|c|}
\hline & Number of lines & $\begin{array}{l}\text { Number of } \\
\text { depressions }\end{array}$ & Number of pits \\
\hline North Ronaldsay $(\mathrm{n}=242)$ & $\begin{array}{c}165 \\
(68 \%)\end{array}$ & $\begin{array}{c}58 \\
(24 \%)\end{array}$ & $\begin{array}{c}19 \\
(8 \%)\end{array}$ \\
\hline Hoy $(n=82)$ & $\begin{array}{c}51 \\
(62 \%)\end{array}$ & $\begin{array}{c}24 \\
(29 \%)\end{array}$ & $\begin{array}{c}7 \\
(9 \%)\end{array}$ \\
\hline Knap of Howar $(n=58)$ & $\begin{array}{c}31 \\
(53 \%)\end{array}$ & $\begin{array}{c}24 \\
(41 \%)\end{array}$ & $\begin{array}{c}3 \\
(6 \%)\end{array}$ \\
\hline Skara Brae $(n=42)$ & $\begin{array}{c}14 \\
(33 \%)\end{array}$ & $\begin{array}{c}23 \\
(55 \%)\end{array}$ & $\begin{array}{c}5 \\
(12 \%)\end{array}$ \\
\hline $\begin{array}{l}\text { Holm of Papa Westray } \\
(\mathrm{n}=25)\end{array}$ & $\begin{array}{c}7 \\
(28 \%)\end{array}$ & $\begin{array}{c}10 \\
(40 \%)\end{array}$ & $\begin{array}{c}8 \\
(32 \%)\end{array}$ \\
\hline
\end{tabular}


Figure 1: A mandible from a modern North Ronaldsay sheep with the lingual surface of the mandibular corpus removed over the three permanent molars. Dashed line indicates the portion of tooth crown hidden within the mandibular bone.

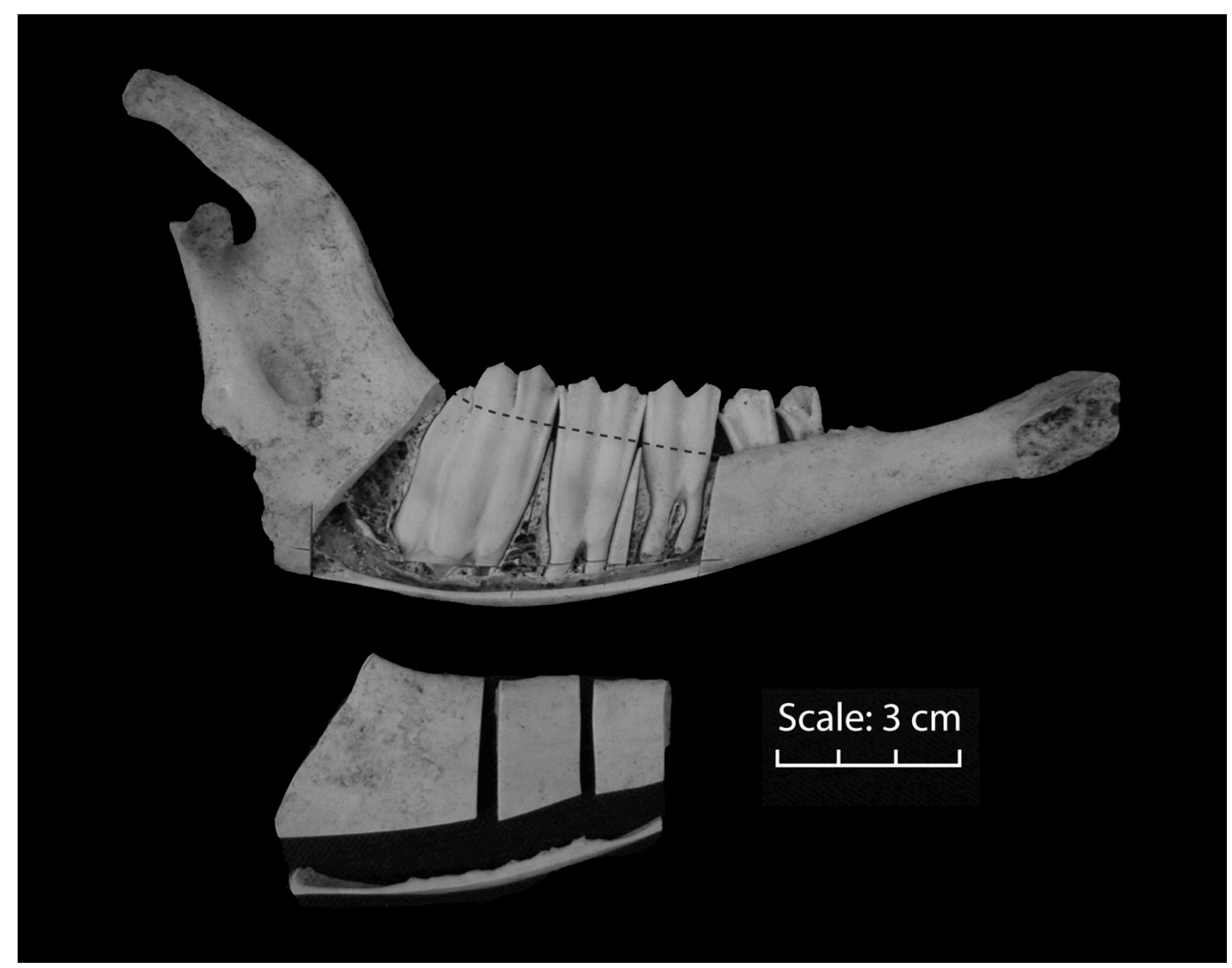


Figure 2: A first molar from North Ronaldsay with a close-up of coronal cementum covering the enamel surface. Dashed line indicates the area where coronal cementum has flaked off, revealing the enamel beneath. Note the presence of pit-type enamel hypoplasia (indicated by arrows) in areas where the coronal cementum is not present.

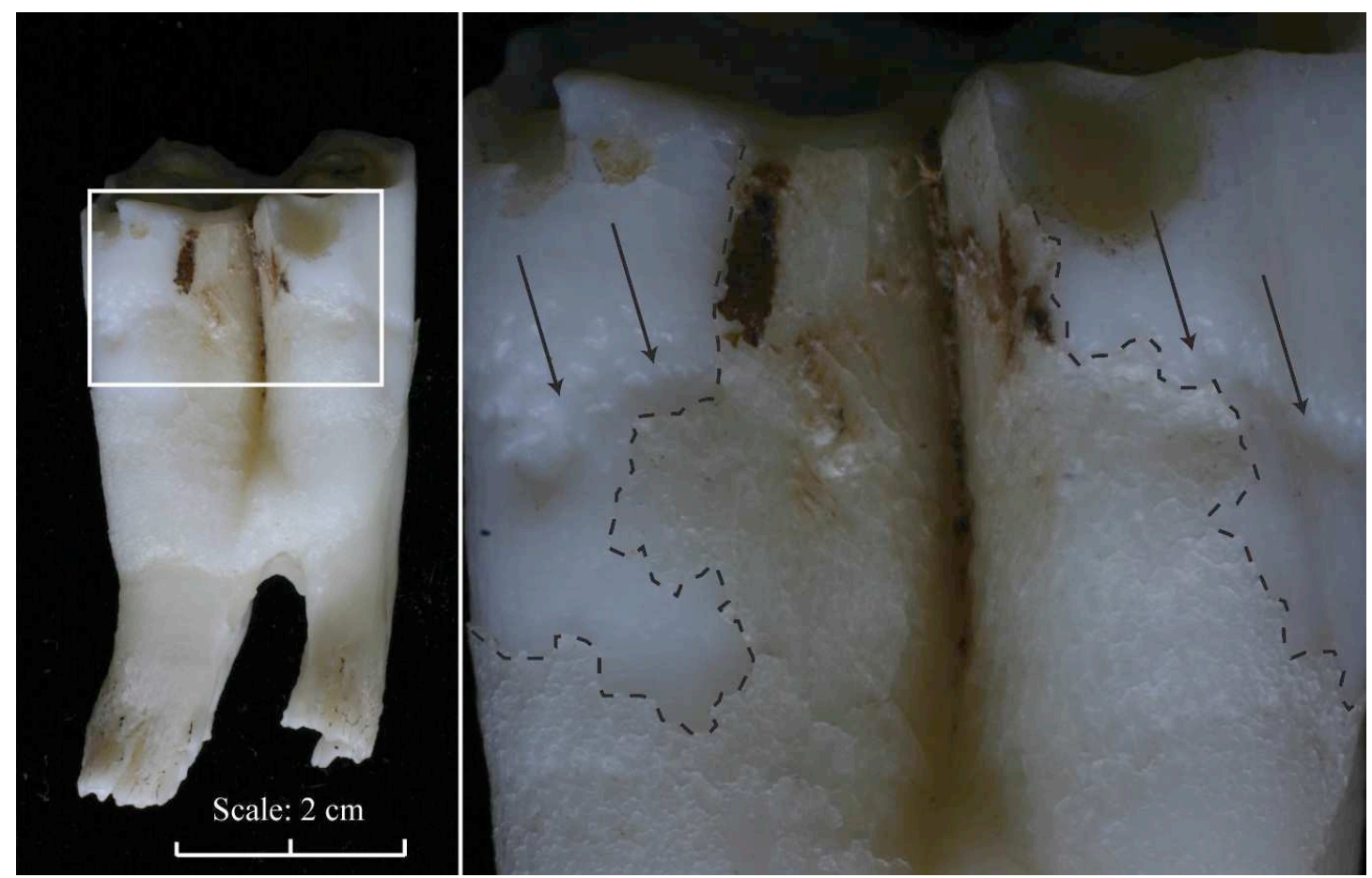


Figure 3: A North Ronaldsay sheep third molar (M3, wear stage 5, 3-4 years of age) with a reconstruction of the early phases of dental wear. In the NR population, the maximum crown height was 38 millimeters. Selective Payne wear stages are shown on the right (numbered 16) correlating to Payne age categories D (1-2 years), E (2-3 years), F (3-4 years), and G (4-6 years) (after Payne 1973). By Payne age category F (3-4 years), only a relatively small amount of this crown has been lost to dental wear (approximately 8 millimeters), meaning that dental wear in this age category will have a minimum impact on recording enamel hypoplasia, which is mainly located on the lower part of the crown.
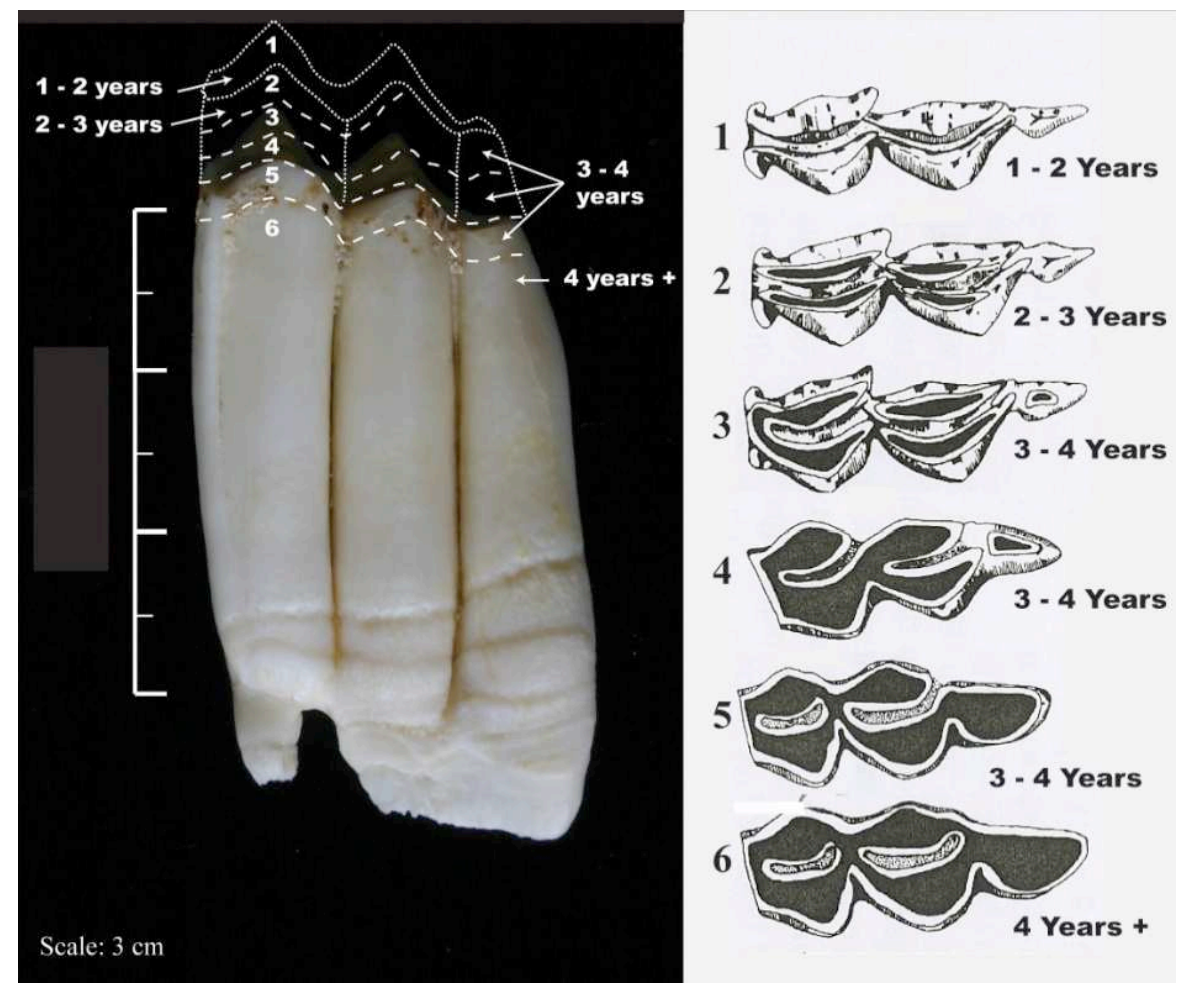
Figure 4: Depression, pit and line type enamel hypoplasia in modern sheep teeth from North Ronaldsay breed.

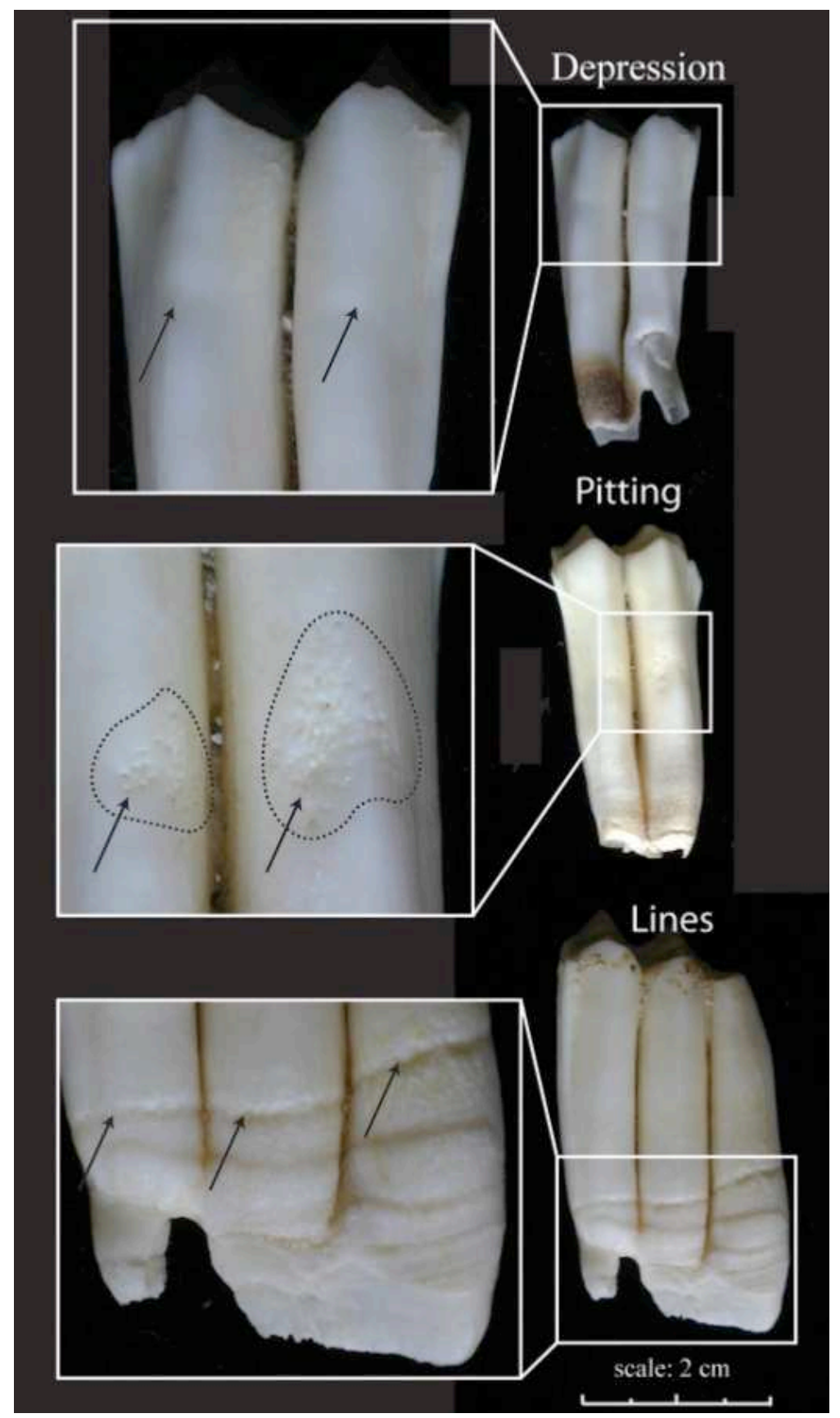


Figure 5: An incomplete first molar (M1) from a North Ronaldsay sheep. Area below the dashed line shows the pitted and porous appearance of poorly mineralised enamel. Black arrows indicate the measurement of enamel hypoplasia in incomplete crowns. The white arrows indicate the measurement of crown height.

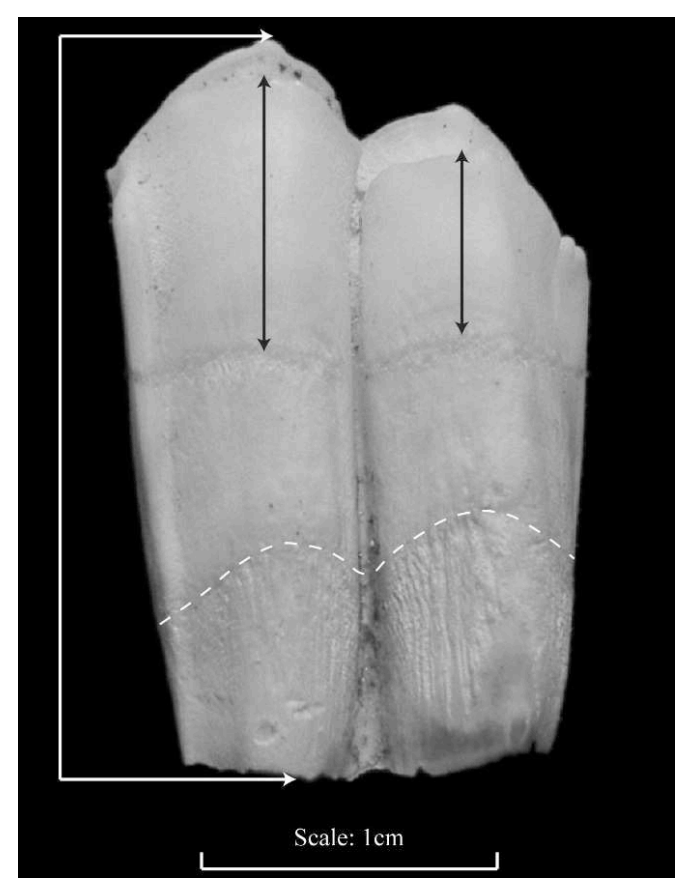


Figure 6: (A) BSE-SEM micrograph of the buccocervical part of a section through the posterior cusp of a left lower 3rd molar of a North Ronaldsay sheep. (B) Macrograph of the buccal view of the tooth, dashed line indicates section plane shown in (A). (C) Macrograph of the lingual view. Cementum coverage of the enamel surface is visible as a thin layer in darker grey in (A). Large white arrowheads indicate the corresponding positions of the macroscopically visible enamel hypoplasia in (A) \& (B) (note presence of additional enamel hypoplasia only visible in the section: small arrows in (A). The hypoplastic defects are more pronounced and thus better visible on the buccal compared to the lingual surface of the tooth. Measurement of enamel hypoplasia location on the buccal surface is indicated by black arrows in (B), measurement of dental crown height is indicated by a black arrow in (C).

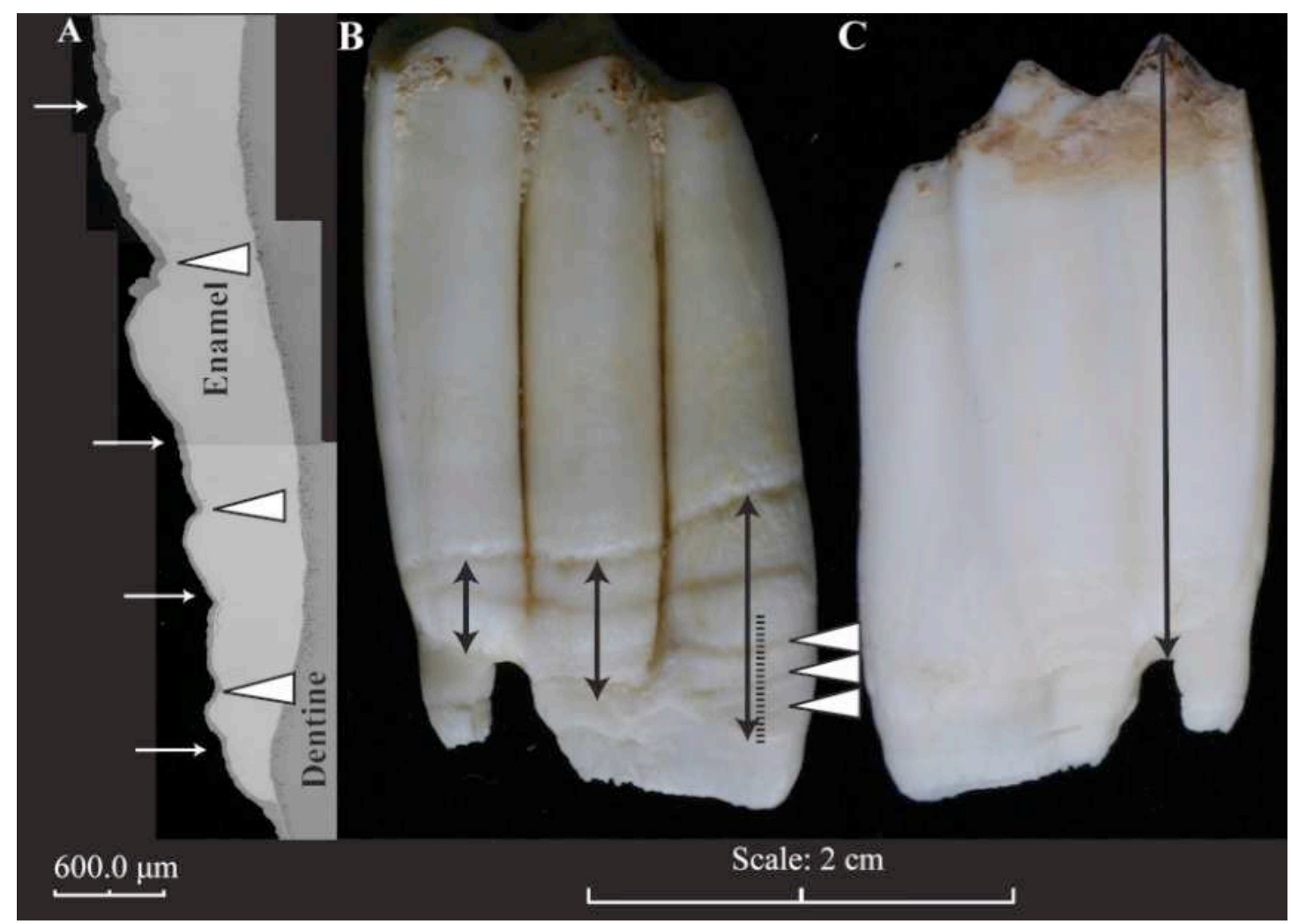


Figure 7: The effect of tooth wear on crown height in Payne age groups $C$ to F (Payne 1973). Only complete teeth are recorded explaining the absence of some age groups. The $\mathrm{X}$ axis represents the height of the tooth crown divided into $2 \mathrm{~mm}$ categories, with the root-enamel junction at 0 and the occlusal surface on the left. The $Y$ axis shows the total number of teeth available for study in each $2 \mathrm{~mm}$ category.

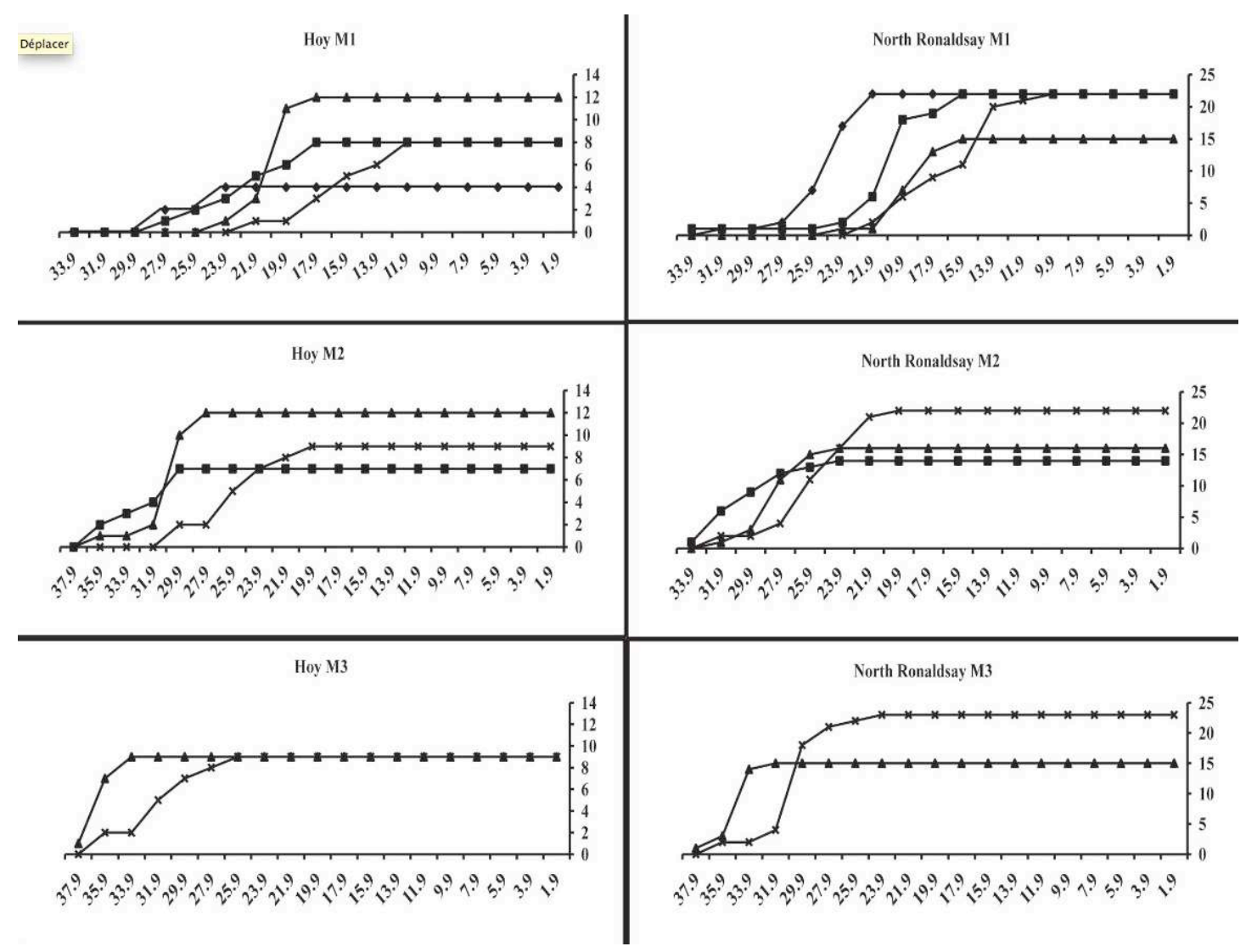


Figure 8: Showing the number of teeth available in each $2 \mathrm{~mm}$ height category and the location of enamel hypoplasia on the tooth crown. This demonstrates that light dental wear does not affect the observation of hypoplasia occurrence. $X$ axis shows the average tooth height tooth crown height in $2 \mathrm{~mm}$ categories with the root-enamel junction at 0 and the occlusal surface on the left, Y axis shows the number of teeth / hypoplasia present in each category.

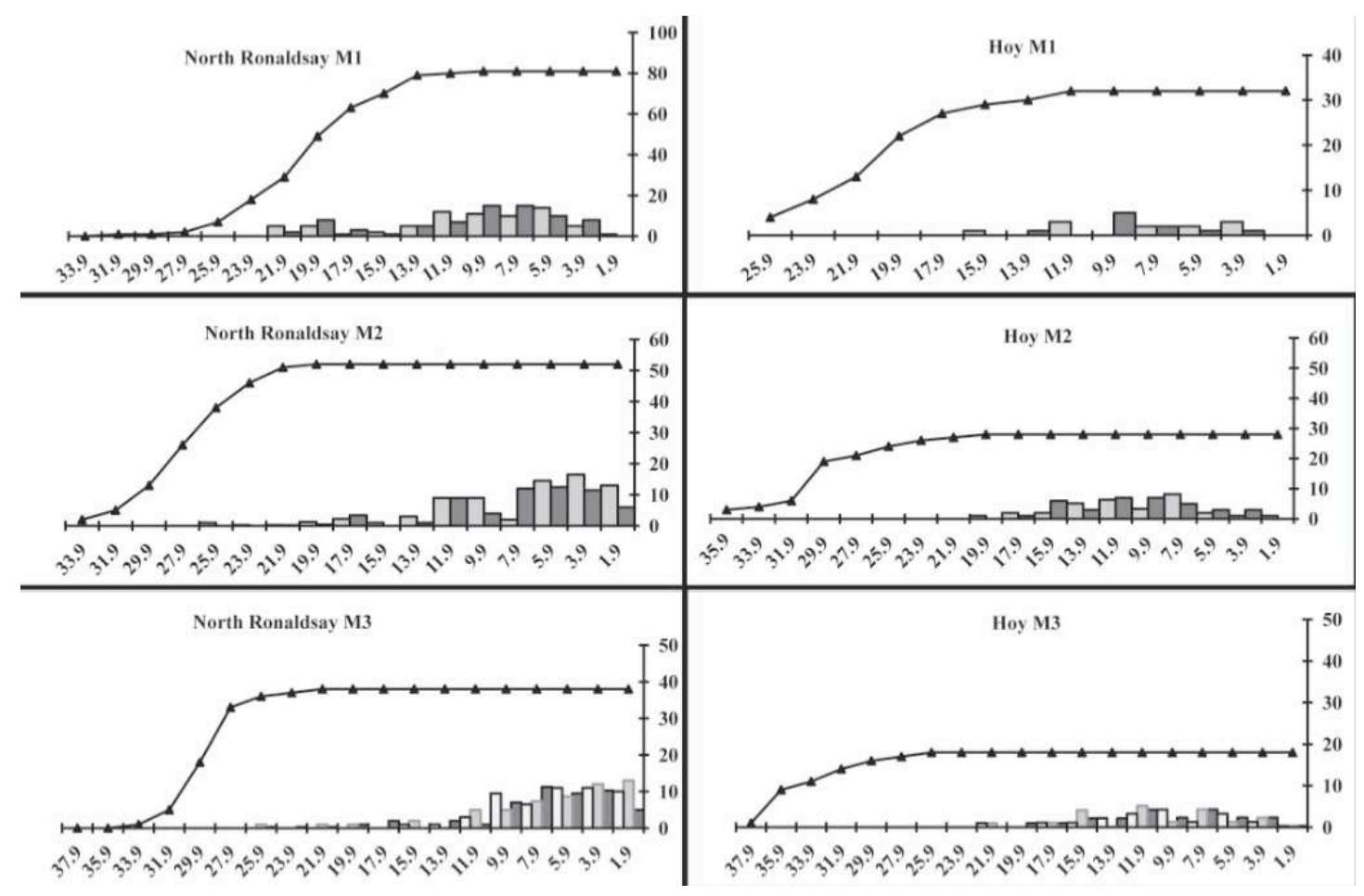


Figure 9: Showing the number of teeth available in each $2 \mathrm{~mm}$ height category and the location of enamel hypoplasia on the tooth crown in the archaeological populations. This demonstrates that light dental wear does not affect the accurate recording of hypoplasia location. $X$ axis shows tooth crown height in $2 \mathrm{~mm}$ categories with the root-enamel junction at 0 and the occlusal surface on the left, $\mathrm{Y}$ axis shows the number of teeth / hypoplasia present in each category.

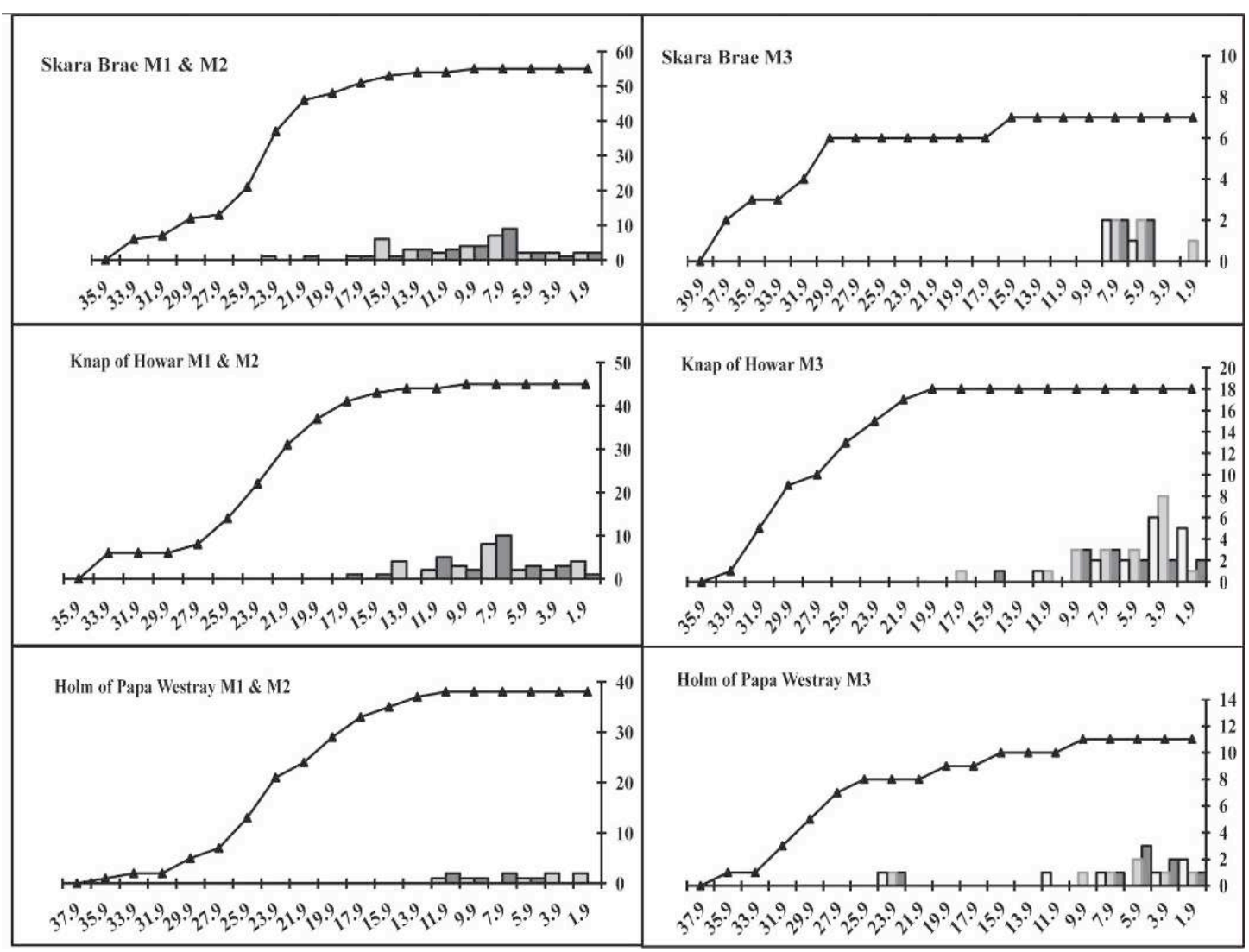

